\title{
A Catalytic One-Pot Synthesis of Indolyl Cyclobutanones
}

\author{
Stefania Porcu \\ Carla Aira Rodriguez \\ Angelo Frongia \\ Francesco Secci* (i)
}

Dipartimento di Scienze Chimiche e Geologiche, Università degli Studi di Cagliari, Complesso Universitario di Monserrato, D block, S.S. 554 09042 Monserrato, Cagliari, Italy

fsecci@unica.it
Received: 14.09 .2020

Accepted after revision: 30.10 .2020

Published online: 03.12 .2020

DOI: 10.1055/s-0040-1706087; Art ID: ss-2020-z0486-fa

Abstract A general strategy for the synthesis of indolyl cyclobutanones via a tandem Brønsted acid catalyzed 2-hydroxycyclobutanone activation-indole nucleophilic addition has been exploited. The procedure leads to a wide range of 2- and 3-functionalized indole derivatives in good to high yields with broad substrate scope.

Key words indoles, cyclobutanones, Brønsted acids, tandem reactions, catalysis

Due to the extremely high number of natural bioactive compounds containing an indole unit, ${ }^{1}$ a wide number of chemoselective syntheses and functionalizations of this heterocycle have been exploited. ${ }^{2}$ In particular, many investigations were focused on the identification of new and specific therapeutic agents for the treatment of several human diseases such as glaucoma, ${ }^{3}$ hypertension, ${ }^{4}$ depression, ${ }^{5}$ Alzheimer's disease, ${ }^{6}$ cardiovascular diseases, ${ }^{7}$ and viral infections. ${ }^{8}$ A number of strained cyclobutyl- and cyclopropylindole derivatives have aroused significant interest, not only for their pharmacological properties but, above all, for their usefulness in drug-receptor affinity studies. Indeed, the affinity of lead compounds can be studied by performing radioligand binding assays using constrained molecular architectures that, by reducing the rotations around certain bonds, clarify the way in which these molecules interact with biological targets. ${ }^{9}$ Examples include compound 4991W93, a 5HT1B/1D receptor and partial agonist with potential for the treatment and prophylaxis of migraine, ${ }^{10}$ the inhibition of leukotriene production, ${ }^{11}$ CRTH2 antagonists $^{12}$ and estrogen receptor modulators ${ }^{13}$ (Scheme $1, \mathrm{a}$ ).
Despite their biological activity, direct insertion of a cyclobutane unit on the indole skeleton is limited to a few multistep approaches, generally requiring the preparation of prefunctionalized substrates (Scheme 1, b).9c,10,14

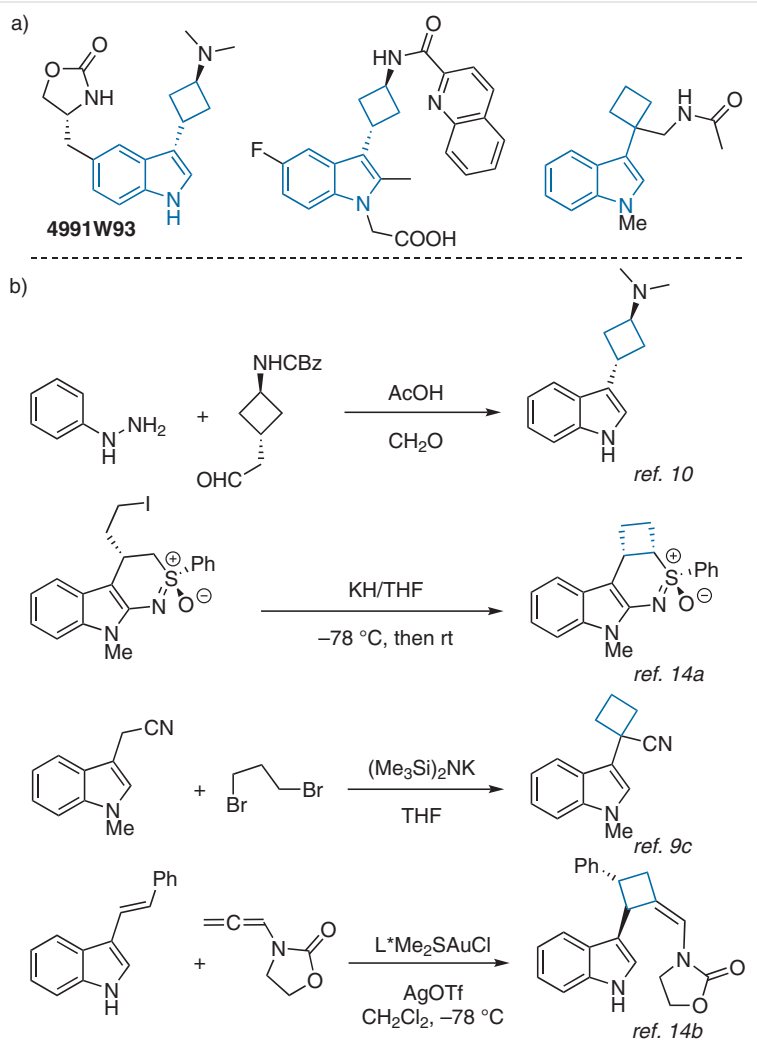

Scheme 1 (a) Representative bioactive cyclobutyl-indole drugs. (b) Strategies for the insertion of a cyclobutanone unit on indoles. 
In this context, a versatile one-pot catalytic and chemoselective procedure would represent a significant advance for drug design and synthesis. We recently reported the preparation of indole cyclopropylcarbaldehydes 3 through a tandem acid-catalyzed addition/ring-contraction reaction of 2-hydroxycyclobutanone (1) and indole (2a) (Scheme 2, a). ${ }^{15}$ Continuing our studies in this research field, ${ }^{16}$ we dis- covered that functionalized aldehyde 3a furnished the corresponding indolylcyclobutanone derivative $\mathbf{4 a}$ on treatment with PTSA at $50{ }^{\circ} \mathrm{C}$ (Scheme 2, b).

This observation inspired us to develop a two-step, onepot synthesis of cyclobutanone indoles $\mathbf{4}$ following the general procedure shown in Scheme 2, c. To explore the feasibility of this process, a series of experiments was performed to identify the best reaction conditions. A summary of the results is reported in Table 1 (see also Table S1 in the

\section{Biographical Sketches}
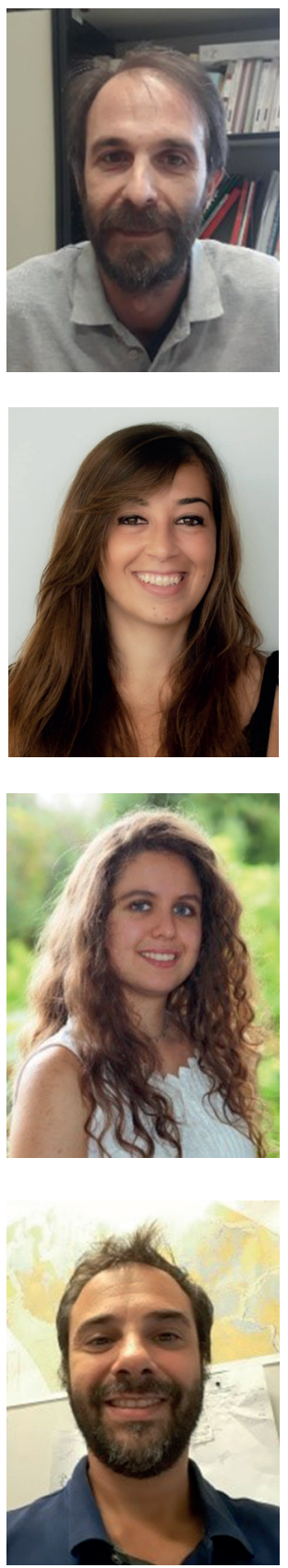

Angelo Frongia was born in Cagliari (Italy) in 1973. He graduated and received his Ph.D. in organic chemistry from the University of Cagliari under the supervision of Prof. P. P. Piras. In 2001, he joined Dr. Jacques Salaün at the Laboratoire des Carbocycles (CNRS) Paris (France) as a postdoctoral fellow. During his studies (2002-

Stefania Porcu obtained her Master's degree from the University of Cagliari (Italy) under the supervision of Prof. Francesco Secci. Since 2017, she has

Carla Aira Rodriguez obtained her Bachelor's degree in chemistry from the University of Vigo (Spain) in 2020. To com-
2008), he completed an internship in the group of Prof. P. P. Piras at the University of Cagliari, and following collaborative postdoctoral research (2009) in the group of Prof. D. J. Aitken at the "Institut de Chimie Moléculaire et des Matériaux d'Orsay', University Paris Sud (France), he started his independent career at the 'Dipartimen-

been a Ph.D. student under the supervision of Prof. Francesco Secci and Prof. Pier Carlo Ricci (Italy). Her research interests include acid-catalyzed synthesis to di Scienze Chimiche e Geologiche' (University of Cagliari) in 2010. He is currently Associate Professor of Organic Chemistry at the Faculty of Sciences. His research interests focus on the synthesis and reactivity of strained carbocycles and asymmetric organocatalytic reactions.

and functionalization of N-heterocycles, their photo-physical characterization and their use as photocatalysts.

plete her M.Sc. degree, she moved to Cagliari and since October 2019 she has worked on the development of novel eco- friendly one-pot functionalizations of indole derivatives under the supervision of Prof. Francesco Secci (Italy).
Francesco Secci was born in Cagliari (Italy) in 1977. He received his Ph.D. in 2006 from the University of Cagliari and Université Paris-Sud (Orsay, France) under the supervision of Prof. P. P. Piras and Dr. J. Ollivier. After a postdoctoral stay (2007-2010) at the University of York (UK) with Prof. P. O'Brien, he moved back to Cagliari to start his own research with a grant from the Fund for Investment in Basic Research (FIRB) (Italy). Since 2013, he has served as a lecturer at the same university, and in 2019 he was promoted to Associate Profes- sor. His research interests are focused on the development of new synthetic methodologies involving strained carbo- and heterocyclic compounds, the study of novel catalytic transformations and their synthetic applications. 
a)

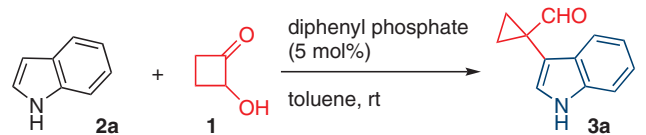

b)

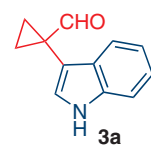

c)

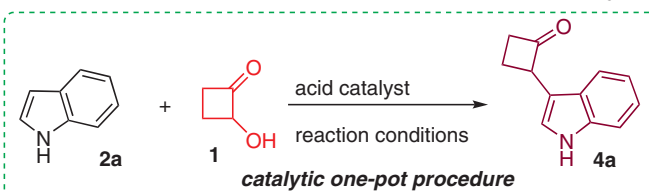

Scheme 2 (a) Acid-catalyzed synthesis of indole cyclopropylcarbaldehyde 3a. (b) The C3-C4 ring expansion of aldehyde 3 a to give cyclobutanone 4a. (c) A catalytic one-pot synthesis of indolyl cyclobutanone derivative $\mathbf{4 a}$.

Supporting Information). We first examined the reaction between 2-hydroxycyclobutanone (1) and indole (2a) in the presence of $\mathrm{TsOH}(10 \mathrm{~mol} \%)$ under neat conditions and isolated the desired product $4 \mathbf{4}$ in only $18 \%$ yield after 24 hours (entry 1 ). The reaction carried out in toluene at $40{ }^{\circ} \mathrm{C}$ gave the product $4 \mathbf{a}$ in an improved $48 \%$ yield (entry 2 ), and this increased to $58 \%$ when the reaction was performed at $50{ }^{\circ} \mathrm{C}$ (entry 3). Increasing the temperature to $60{ }^{\circ} \mathrm{C}$ did not lead to any further improvement in the yield of $\mathbf{4 a}$ (entry 4 ). Furthermore, no significant benefits were obtained from the use of $3 \AA$ molecular sieves or other anhydrous reaction conditions. Finally, a catalyst screen using recyclable NR-50
(Nafion resin 50), AR-15 (Amberlyst resin 15) and AR-35 (Amberlyst resin 35) (entries 5, 6 and 7) showed that the optimum yield of $4 \mathbf{a}(96 \%)$ was obtained when using AR-15 (10 mol\% loading) at $50{ }^{\circ} \mathrm{C}$ in toluene.

With optimized reaction conditions in hand, we next studied the scope of this one-pot, two-step procedure using substituted indoles 2a-v (Scheme 3). Good functional group tolerance was observed for indoles $\mathbf{2}$, allowing access to a variety of cyclobutanone adducts $\mathbf{4}$. Indoles $\mathbf{2 a - d}$ furnished the corresponding adducts $\mathbf{4 a - d}$ in good to high yields (73-96\%). Satisfactory results were achieved by reacting 2-hydroxycyclobutanone (1) with $\mathrm{N}$-substituted indoles $2 \mathbf{e}-\mathbf{i}$, affording the cyclobutanones $\mathbf{4 e -} \mathbf{i}$ in $89-94 \%$ yields. ${ }^{17}$ The 2 - and 3 -alkyl/aryl indoles $\mathbf{2 j - q}$ gave good conversions and yields of the corresponding indolyl ketones $\mathbf{4 j} \mathbf{j} \mathbf{p}$, with $\mathbf{4 q}$ being recovered as a 50.50 mixture of diastereoisomers ( $90 \%$ yield), probably due to epimerization at C2 of the cyclobutanone moiety during the chromatographic purification step. On the other hand, reactions of $\mathbf{1}$ with indoles $2 \mathbf{r}-\mathbf{v}$, bearing electron-withdrawing groups at the C4 or $\mathrm{C} 5$ positions, did not occur as desired. In these cases, cyclopropyl carbaldehydes $\mathbf{3 r}-\mathbf{v}$ were isolated as unique reaction products in yields of $74-90 \%$.

Further experiments carried out with $N$-benzylpyrrole afforded the corresponding cyclopropanes $\mathbf{3 w} / \mathbf{3} \mathbf{w}^{\mathbf{\prime}}$ (as an $80: 20$ mixture of regioisomers) in $68 \%$ overall yield. The reaction of 1 with 3,5-dimethoxyphenol yielded the cyclobutanone $\mathbf{4 x}$ in $86 \%$ yield.

The results obtained during this study allowed us to put forward a plausible mechanism for the acid-catalyzed onepot addition of a cyclobutanone to the indole skeleton

Table 1 Initial Screening Studies ${ }^{\mathrm{a}}$

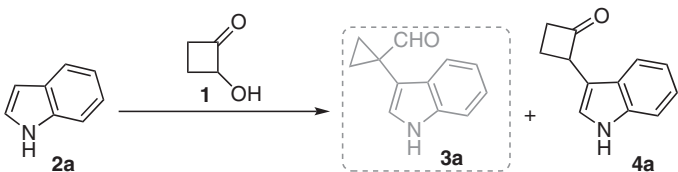

\begin{tabular}{|c|c|c|c|c|c|c|}
\hline Entry & Catalyst (mol\%) & Solvent & Temp $\left({ }^{\circ} \mathrm{C}\right)$ & Ratio of $3 a / 4 a^{b}$ & Conv. $(\%)^{c}$ & Yield (\%) \\
\hline 1 & $\mathrm{TsOH}(10)$ & neat & 40 & $2: 1$ & 21 & 18 \\
\hline 2 & $\mathrm{TsOH}(10)$ & toluene & 40 & $1: 1$ & 50 & 48 \\
\hline 3 & $\mathrm{TsOH}(10)$ & toluene & 50 & $1: 3$ & 63 & 58 \\
\hline 4 & $\mathrm{TsOH}(10)$ & toluene & 60 & 1:16 & 60 & 54 \\
\hline 5 & NR-50 (10) & toluene & 50 & $1: 22$ & 96 & 94 \\
\hline 6 & AR-15 (10) & toluene & 50 & $1: 28$ & 97 & 96 \\
\hline 7 & AR-35 (10) & toluene & 50 & $1: 28$ & 93 & 90 \\
\hline
\end{tabular}

a Reaction conditions: 1 (50 mg, $0.58 \mathrm{mmol}$ ), 2 a (1.0 equiv), acid catalyst [Nafion resin 50 (NR-50), Amberlyst resin 15 (AR-15) or Amberlyst resin 35 (AR-35): 10 mol\%], solvent $(1.5 \mathrm{~mL}), 8-24 \mathrm{~h}, 40-50^{\circ} \mathrm{C}$.

b The 3a:4a ratio was determined by ${ }^{1} \mathrm{H}$ NMR analysis.

c The conversion into $4 \mathrm{a}$ was determined by ${ }^{1} \mathrm{H}$ NMR analysis.

d Yield of isolated product 4a after flash chromatography. 


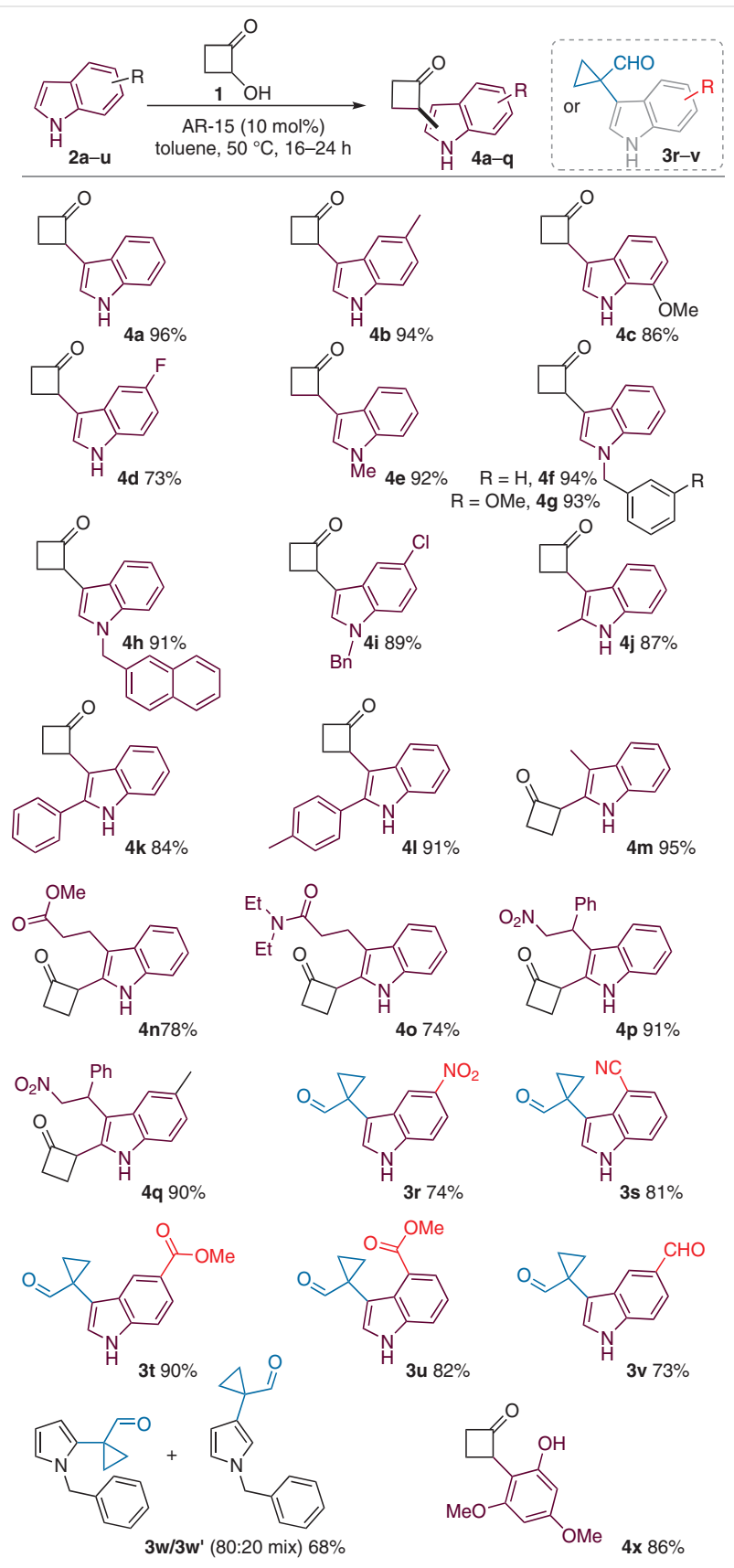

Scheme 3 Exploration of the substrate scope

(Scheme 4). In our hypothesis, the protonation of cyclobutanone 1 promotes indole nucleophilic attack leading to the formation of the adduct $\mathbf{I}^{15,16 a, b}$ At room temperature, this intermediate undergoes a rapid C4-C3 ring contraction via carbocationic species II, mainly affording the cyclopropyl carbaldehydes $3 .{ }^{16}$ At higher temperatures $\left(50{ }^{\circ} \mathrm{C}\right)$, the establishment of an equilibrium between the three-membered-ring species $\mathbf{3}$ and the cyclobutane carbocationic intermediate II leads to the formation of derivatives $\mathbf{4} .^{18,19}$
However, this process is favored by the presence of electron-donating groups on the heterocyclic system. On the other hand, reactions carried out with indoles bearing electron-withdrawing groups (e.g., $2 \mathbf{r}-\mathbf{v}$ ) destabilize the carbocationic species II and promote the C4-C3 ring contraction as a preferential stabilization process. ${ }^{19,20}$

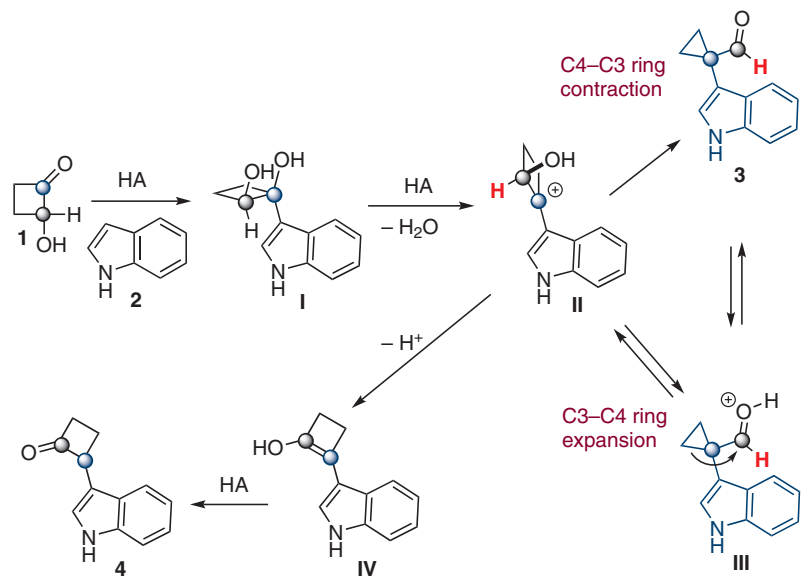

Scheme 4 Proposed reaction mechanism for the synthesis of cyclobutanones $\mathbf{4}$ and cyclopropyl carbaldehydes $\mathbf{3}$ under acid-catalyzed conditions

In summary, a new one-pot catalytic functionalization of indoles has been established under mild conditions. This method provides a straightforward access to diverse indolyl cyclobutanone compounds in good to excellent yields, underlining a broad substrate scope and some limitations related to the presence of electron-withdrawing groups. This two-step, one-pot process has been studied by using a recyclable polystyrene-based ion exchange resin. Further studies aimed toward adapting this reaction to a continuousflow process are currently underway in our laboratories.

Diphenyl phosphate, Amberlyst 15, Amberlyst 35 and indoles $\mathbf{2 a}, \mathbf{d}, \mathbf{i}, \mathbf{l}, \mathbf{q}, \mathbf{r}$ were purchased from Sigma-Aldrich and used without further purification. Indoles $\mathbf{2 b}, \mathbf{c}, \mathbf{m}, \mathbf{s}$ were purchased from TCI and used without further purification. Unless noted otherwise, all experiments were carried out in a $5 \mathrm{~mL}$ vial closed with a screw cap and equipped with a magnetic stir bar. Analytical thin-layer chromatography was performed using $0.25 \mathrm{~mm}$ Aldrich silica gel 60-F plates. Flash chromatography was performed using Merck silica gel (70-200 mesh). Yields refer to chromatographically and spectroscopically pure materials. Melting points were determined with a Büchi M-560 apparatus. Infrared spectra were recorded on FT-IR Bruker Equinox-55 or Thermo Fisher Scientific Nicolet IS50 FTIR spectrophotometers and are reported in wavenumbers $\left(\mathrm{cm}^{-1}\right) .{ }^{1} \mathrm{H}$ and ${ }^{13} \mathrm{C}$ NMR spectra were obtained on Bruker DRX $600\left({ }^{1} \mathrm{H}, 600 \mathrm{MHz} ;{ }^{13} \mathrm{C}, 150 \mathrm{MHz}\right)$ or Varian 500 $\left({ }^{1} \mathrm{H}, 500 \mathrm{MHz} ;{ }^{13} \mathrm{C}, 126 \mathrm{MHz}\right)$ spectrometers at $27{ }^{\circ} \mathrm{C}$ using $\mathrm{CDCl}_{3}$ (internal reference $=7.26 \mathrm{ppm}$ ) as the solvent. ${ }^{13} \mathrm{C}$ NMR were recorded at $126 \mathrm{MHz}$ (internal reference $=77.00 \mathrm{ppm}$ ) using $\mathrm{CDCl}_{3}$ as the solvent. Chemical shifts $(\delta)$ are given in ppm. Coupling constants $(J)$ are reported in $\mathrm{Hz}$. Low-resolution mass spectrometry was performed 
using an Agilent-HP GC-MS (EI, $70 \mathrm{eV}$ ). High-resolution mass spectrometry (HRMS) was performed in fast atom bombardment (FAB+) ionization mode (ESI) using Bruker micrOTF-Q II and/or Agilent QTOF 6520 instruments.

\section{Cyclobutyl Indoles 4; General Procedure}

In a $5 \mathrm{~mL}$ glass vial, a mixture of 2-hydroxycyclobutanone (1a) (100 $\mathrm{mg}, 1.1 \mathrm{mmol})$, indole $\mathbf{2 a}-\mathbf{v}(1.0 \mathrm{mmol})$, Amberlyst 15 (10 mol\%) and toluene $(3.0 \mathrm{~mL})$ was stirred at $50{ }^{\circ} \mathrm{C}$. After completion of the reaction, the crude product was loaded on a silica gel column and purified by flash chromatography using hexanes/diethyl ether (10:1 to $3: 1)$ as the eluent.

\section{2-(1H-Indol-3-yl)cyclobutanone (4a)}

Yield: $117 \mathrm{mg}$ (96\%); colorless oil.

${ }^{1} \mathrm{H} \mathrm{NMR}\left(500 \mathrm{MHz}, \mathrm{CDCl}_{3}\right): \delta=7.97(\mathrm{~s}, 1 \mathrm{H}), 7.50(\mathrm{~d}, J=7.8 \mathrm{~Hz}, 1 \mathrm{H})$, $7.29(\mathrm{~d}, J=8.1 \mathrm{~Hz}, 1 \mathrm{H}), 7.17-7.11(\mathrm{~m}, 1 \mathrm{H}), 7.08(\mathrm{~d}, J=1.4 \mathrm{~Hz}, 1 \mathrm{H})$, 7.06 (td, $J=7.6,1.0 \mathrm{~Hz}, 1 \mathrm{H}), 4.68$ (tdd, $J=7.9,2.5,1.2 \mathrm{~Hz}, 1 \mathrm{H}$ ), 3.31$3.14(\mathrm{~m}, 1 \mathrm{H}), 3.07$ (dddd, $J=17.6,9.7,4.9,2.7 \mathrm{~Hz}, 1 \mathrm{H}), 2.55$ (qd, $J=$ $10.6,4.9 \mathrm{~Hz}, 1 \mathrm{H}$ ), 2.14 (ddt, $J=11.0,9.6,8.1 \mathrm{~Hz}, 1 \mathrm{H}$ ).

${ }^{13} \mathrm{C}$ NMR $\left(126 \mathrm{MHz}, \mathrm{CDCl}_{3}\right): \delta=208.8,136.3,126.3,122.4,121.1$, 119.7, 119.0, 112.0, 111.2, 57.0, 45.1, 18.3.

All analytical data were in good accordance with reported data. ${ }^{16}$

\section{2-(5-Methyl-1H-indol-3-yl)cyclobutanone (4b)}

Yield: $187 \mathrm{mg}$ (94\%); colorless oil.

FTIR (film): 2994, 1788, 1612, 1454, 1228, 1129, $1011 \mathrm{~cm}^{-1}$.

${ }^{1} \mathrm{H} \mathrm{NMR}\left(500 \mathrm{MHz}, \mathrm{CDCl}_{3}\right.$ ): $\delta=7.94(\mathrm{~s}, 1 \mathrm{H}), 7.33(\mathrm{~s}, 1 \mathrm{H}), 7.25$ (d, $J=$ $10.0 \mathrm{~Hz}, 1 \mathrm{H}), 7.11$ (d, $J=1.4 \mathrm{~Hz}, 1 \mathrm{H}), 7.03(\mathrm{~d}, J=8.3 \mathrm{~Hz}, 1 \mathrm{H}), 4.80-$ $4.60(\mathrm{~m}, 1 \mathrm{H}), 3.32-3.23(\mathrm{~m}, 1 \mathrm{H}), 3.13$ (dddd, $J=17.6,9.6,4.9,2.7 \mathrm{~Hz}$, $1 \mathrm{H}$ ), 2.61 (ddd, $J=21.4,10.6,4.9 \mathrm{~Hz}, 1 \mathrm{H}$ ), 2.45 (s, $3 \mathrm{H}$ ), 2.29-2.13 (m, $1 \mathrm{H})$.

${ }^{13} \mathrm{C}$ NMR $\left(126 \mathrm{MHz}, \mathrm{CDCl}_{3}\right): \delta=208.8,136.3,126.3,122.4,121.1$, 119.7, 119.0, 112.0, 111.2, 57.0, 45.1, 18.3.

HRMS (ESI): $m / z$ [M $+\mathrm{Na}]^{+}$calcd for $\mathrm{C}_{13} \mathrm{H}_{13} \mathrm{NNaO}$ : 222.0895; found: 222.0898 .

\section{2-(7-Methoxy-1H-indol-3-yl)cyclobutanone (4c)}

Yield: $185 \mathrm{mg}(86 \%)$; green oil.

FTIR (film): 2998, 1789, 1614, 1460, 1221, 1129, $1009 \mathrm{~cm}^{-1}$.

${ }^{1} \mathrm{H}$ NMR $\left(500 \mathrm{MHz}, \mathrm{CDCl}_{3}\right): \delta=8.12(\mathrm{~s}, 1 \mathrm{H}), 7.08(\mathrm{~s}, 1 \mathrm{H}), 7.00(\mathrm{~d}, J=$ $7.0 \mathrm{~Hz}, 1 \mathrm{H}), 6.87(\mathrm{~d}, J=7.0 \mathrm{~Hz}, 1 \mathrm{H}), 6.59(\mathrm{dd}, J=6.5,3.5 \mathrm{~Hz}, 1 \mathrm{H}), 4.90$ $(\mathrm{t}, J=6.5 \mathrm{~Hz}, 1 \mathrm{H}), 3.80(\mathrm{~s}, 3 \mathrm{H}), 2.72-2.67(\mathrm{~m}, 1 \mathrm{H}), 2.40-2.37(\mathrm{~m}, 1 \mathrm{H})$, 2.08-2.01 (m, $2 \mathrm{H})$.

${ }^{13} \mathrm{C}$ NMR $\left(126 \mathrm{MHz}, \mathrm{CDCl}_{3}\right): \delta=210.9,154.4,131.3,128.3,124.8$, $116.5,112.6,112.1,100.6,55.9,40.8,29.1,19.0$.

HRMS (ESI): $m / z[\mathrm{M}+\mathrm{Na}]^{+}$calcd for $\mathrm{C}_{13} \mathrm{H}_{13} \mathrm{NNaO}_{2}: 238.0844$; found: 238.0851 .

\section{2-(5-Fluoro-1H-indol-3-yl)cyclobutanone (4d)}

Yield: $148 \mathrm{mg}$ (73\%); yellow oil.

FTIR (film): 2986, 1790, 1463, 1227, 1145, $986 \mathrm{~cm}^{-1}$.

${ }^{1} \mathrm{H}$ NMR (500 MHz, $\mathrm{CDCl}_{3}$ ): $\delta=7.97$ (br s, $\left.1 \mathrm{H}\right), 7.22-7.19(\mathrm{~m}, 1 \mathrm{H})$, $7.12(\mathrm{td}, J=9.8,2.1 \mathrm{~Hz}, 2 \mathrm{H}), 6.88(\mathrm{td}, J=9.1,2.3 \mathrm{~Hz}, 1 \mathrm{H}), 4.62(\mathrm{t}, J=$ $9.2 \mathrm{~Hz}, 1 \mathrm{H}), 3.30-3.12(\mathrm{~m}, 1 \mathrm{H}), 3.14-2.93(\mathrm{~m}, 1 \mathrm{H}), 2.55$ (qd, $J=10.7$, $5.0 \mathrm{~Hz}, 1 \mathrm{H}), 2.17-2.00(\mathrm{~m}, 1 \mathrm{H})$.
${ }^{13} \mathrm{C}$ NMR $\left(126 \mathrm{MHz}, \mathrm{CDCl}_{3}\right): \delta=208.7,129.7,122.9,111.9$ (d, $J=1.8$ $\mathrm{Hz}), 111.8,110.9$ (d, $J=1.9 \mathrm{~Hz}), 110.7,104.1$ (d, $J=1.8 \mathrm{~Hz}), 104.0,56.8$, 45.1, 18.2.

HRMS (ESI): $m / z$ [M $+\mathrm{Na}]^{+}$calcd for $\mathrm{C}_{12} \mathrm{H}_{10} \mathrm{FNNaO}$ : 226.0644; found: 226.0651 .

\section{2-(1-Methyl-1H-indol-3-yl)cyclobutanone (4e)}

Yield: $183 \mathrm{mg}$ (92\%); orange oil.

All analytical data were in good accordance with reported data. ${ }^{15}$

\section{2-(1-Benzyl-1H-indol-3-yl)cyclobutanone (4f)}

Yield: $258 \mathrm{mg}$ (94\%); yellow oil.

${ }^{1} \mathrm{H}$ NMR $\left(500 \mathrm{MHz}, \mathrm{CDCl}_{3}\right): \delta=7.49(\mathrm{~d}, J=7.9 \mathrm{~Hz}, 1 \mathrm{H}), 7.22(\mathrm{dd}, J=$ 11.7, $4.2 \mathrm{~Hz}, 3 \mathrm{H}$ ), 7.18 (d, J = 8.4 Hz, $2 \mathrm{H}$ ), 7.06-7.03 (m, $3 \mathrm{H}), 7.00$ (s, 1 H), $5.18(\mathrm{~s}, 2 \mathrm{H}), 4.67(\mathrm{td}, J=8.4,2.0 \mathrm{~Hz}, 1 \mathrm{H}), 3.29-3.12(\mathrm{~m}, 1 \mathrm{H}), 3.04$ (dddd, $J=17.5,9.6,4.9,2.7 \mathrm{~Hz}, 1 \mathrm{H}$ ), 2.53 (qd, $J=10.6,4.9 \mathrm{~Hz}, 1 \mathrm{H}$ ), 2.22-1.95 ( $\mathrm{m}, 1 \mathrm{H})$.

${ }^{13} \mathrm{C}$ NMR $\left(126 \mathrm{MHz}, \mathrm{CDCl}_{3}\right): \delta=202.1,137.3,136.7,128.7,127.6$, 126.8, 125.2, 122.1, 119.7, 119.4, 119.2, 111.0, 109.8, 57.0, 50.0, 45.1, 18.4.

All analytical data were in good accordance with reported data. ${ }^{16}$

\section{2-[1-(3-Methoxybenzyl)-1H-indol-3-yl]cyclobutanone (4g)}

Yield: $283 \mathrm{mg}$ (93\%); colorless oil.

FTIR (film): 2984, 1789, 1458, 1227, 1144, $908 \mathrm{~cm}^{-1}$.

${ }^{1} \mathrm{H}$ NMR (600 MHz, $\mathrm{CDCl}_{3}$ ): $\delta=7.49(\mathrm{~d}, J=7.9 \mathrm{~Hz}, 1 \mathrm{H}), 7.18(\mathrm{~s}, 1 \mathrm{H})$, 7.15-7.08 (m, $2 \mathrm{H}), 7.03$ (dd, $J=11.0,3.9 \mathrm{~Hz}, 1 \mathrm{H}), 7.00(\mathrm{~s}, 1 \mathrm{H}), 6.71$ (dd, $J=8.2,2.4 \mathrm{~Hz}, 1 \mathrm{H}), 6.63(\mathrm{~d}, J=7.6 \mathrm{~Hz}, 1 \mathrm{H}), 6.59(\mathrm{~s}, 1 \mathrm{H}), 5.15(\mathrm{~s}, 2$ H), 4.74-4.57 (m, $1 \mathrm{H}$ ), 3.66 (s, $3 \mathrm{H}$ ), 3.19 (dddd, $J=18.0,10.7,8.3,2.5$ $\mathrm{Hz}, 1 \mathrm{H}$ ), 3.04 (dddd, $J=17.5,9.6,4.9,2.7 \mathrm{~Hz}, 1 \mathrm{H}), 2.53$ (qd, $J=10.7$, $4.9 \mathrm{~Hz}, 1 \mathrm{H}), 2.12$ (dtd, J = 10.9, 9.6, $8.2 \mathrm{~Hz}, 1 \mathrm{H}$ ).

${ }^{13} \mathrm{C}$ NMR $\left(151 \mathrm{MHz}, \mathrm{CDCl}_{3}\right): \delta=208.9,159.9,138.9,136.8,129.8$, $127.0,125.2,122.1,119.4,119.2,119.1,112.7,112.7,111.0,109.8$, $57.0,55.1,49.9,45.0,18.4$.

HRMS (ESI): $m / z$ [M + Na] $]^{+}$calcd for $\mathrm{C}_{20} \mathrm{H}_{19} \mathrm{NNaO}_{2}: 328.1313$; found: 328.1314 .

2-[1-(Naphthalen-2-ylmethyl)-1H-indol-3-yl]cyclobutanone (4h) Yield: $295 \mathrm{mg}$ (91\%); brown solid; $\mathrm{mp} 76{ }^{\circ} \mathrm{C}$.

FTIR (film): 2952, 1790, 1612, 1463, 1352, 1200, $1072 \mathrm{~cm}^{-1}$.

${ }^{1} \mathrm{H} \mathrm{NMR}\left(500 \mathrm{MHz}, \mathrm{CDCl}_{3}\right): \delta=7.77(\mathrm{~m}, 3 \mathrm{H}), 7.61-7.55(\mathrm{~m}, 2 \mathrm{H}), 7.48-$ 7.40 (m, $2 \mathrm{H}), 7.29$ (d, $J=8.1 \mathrm{~Hz}, 1 \mathrm{H}), 7.26-7.21(\mathrm{~m}, 1 \mathrm{H}), 7.20-7.14$ (m, $1 \mathrm{H}), 7.13-7.08$ (m, $2 \mathrm{H}), 5.39$ (s, $2 \mathrm{H}), 4.80-4.67$ (m, $1 \mathrm{H}), 3.25$ (dddd, $J=17.8,10.6,8.3,2.5 \mathrm{~Hz}, 1 \mathrm{H}$ ), 3.11 (dddd, $J=17.5,9.6,4.9,2.7$ $\mathrm{Hz}, 1 \mathrm{H}$ ), 2.60 (qd, $J=10.6,4.9 \mathrm{~Hz}, 1 \mathrm{H}), 2.19$ (ddt, $J=10.9,9.6,8.2 \mathrm{~Hz}$, $1 \mathrm{H})$.

${ }^{13} \mathrm{C}$ NMR $\left(126 \mathrm{MHz}, \mathrm{CDCl}_{3}\right): \delta=208.8,136.9,134.7,133.3,132.8$, 128.6, 127.8, 127.6, 127.1, 126.3, 126.0, 125.6, 125.2, 124.8, 122.1, $119.4,119.3,111.1,109.8,57.0,50.2,45.0,18.4$.

HRMS (ESI): $m / z[\mathrm{M}+\mathrm{Na}]^{+}$calcd for $\mathrm{C}_{23} \mathrm{H}_{19} \mathrm{NNaO}$ : 348.1364; found: 348.1371 .

\section{2-(1-Benzyl-5-chloro-1H-indol-3-yl)cyclobutanone (4i)}

Yield: $275 \mathrm{mg}$ (89\%); yellow oil.

FTIR (film): 3073, 1783, 1441, 1224, 1018, $990 \mathrm{~cm}^{-1}$. 
${ }^{1} \mathrm{H}$ NMR $\left(500 \mathrm{MHz}, \mathrm{CDCl}_{3}\right): \delta=7.44(\mathrm{~d}, J=1.3 \mathrm{~Hz}, 1 \mathrm{H}), 7.20(\mathrm{dd}, J=$ 10.6, 7.1 Hz, $3 \mathrm{H}$ ), 7.05 (t, J = 7.8 Hz, $2 \mathrm{H}$ ), 7.04-6.99 (m, $3 \mathrm{H}$ ), 5.14 (s, 2 H), 4.59 (td, $J=8.1,2.2 \mathrm{~Hz}, 1 \mathrm{H}$ ), 3.19 (dddd, $J=18.9,10.6,8.4,2.4 \mathrm{~Hz}$, $1 \mathrm{H}), 3.09-2.92(\mathrm{~m}, 1 \mathrm{H}), 2.52$ (qd, $J=10.7,4.9 \mathrm{~Hz}, 1 \mathrm{H}), 2.07$ (tt, $J=$ $10.8,8.3 \mathrm{~Hz}, 1 \mathrm{H})$.

${ }^{13} \mathrm{C}$ NMR $\left(126 \mathrm{MHz}, \mathrm{CDCl}_{3}\right): \delta=208.4,137.0,135.3,129.3,128.9$, $128.2,127.9,127.1,126.9,126.7,125.4,122.6,118.9,111.0,110.9$, $56.8,50.4,45.3,18.5$.

HRMS (ESI): $m / z$ [M + Na $]^{+}$calcd for $\mathrm{C}_{19} \mathrm{H}_{16} \mathrm{ClNNaO}$ : 332.0818; found: 332.0821 .

\section{2-(2-Methyl-1H-indol-3-yl)cyclobutanone (4j)}

Yield: $173 \mathrm{mg}(87 \%)$; yellow oil.

FTIR (film) : 2989, 1788, 1457, 1231, $1144 \mathrm{~cm}^{-1}$.

${ }^{1} \mathrm{H} \mathrm{NMR}\left(500 \mathrm{MHz}, \mathrm{CDCl}_{3}\right): \delta=7.83($ br s, $1 \mathrm{H}), 7.33(\mathrm{~d}, J=7.8 \mathrm{~Hz}, 1 \mathrm{H}$ ), $7.25(\mathrm{~d}, J=6.5 \mathrm{~Hz}, 1 \mathrm{H}), 7.10(\mathrm{t}, J=7.1 \mathrm{~Hz}, 1 \mathrm{H}), 7.08-7.01(\mathrm{~m}, 1 \mathrm{H})$, 4.67 (ddt, $J=10.7,8.6,2.2 \mathrm{~Hz}, 1 \mathrm{H}$ ), 3.28 (dddd, $J=19.3,10.5,8.7,2.0$ $\mathrm{Hz}, 1 \mathrm{H}$ ), 3.23-3.12 (m, $1 \mathrm{H}$ ), 2.52 (qd, $J=10.8,4.8 \mathrm{~Hz}, 1 \mathrm{H}$ ), 2.40-2.34 (m, $1 \mathrm{H}), 2.33(\mathrm{~s}, 3 \mathrm{H})$.

${ }^{13} \mathrm{C}$ NMR $\left(126 \mathrm{MHz}, \mathrm{CDCl}_{3}\right): \delta=210.1,135.3,131.9,127.3,121.3$, 119.5, 118.1, 110.4, 107.2, 57.0, 45.0, 18.3, 12.1 .

HRMS (ESI): $m / z$ [M $+\mathrm{Na}]^{+}$calcd for $\mathrm{C}_{13} \mathrm{H}_{13} \mathrm{NNaO}$ : 222.0895; found: 222.0899 .

\section{2-(2-Phenyl-1H-indol-3-yl)cyclobutanone (4k)}

Yield: $219 \mathrm{mg}$ (84\%); colorless oil.

FTIR (film): 3054, 2989, 1789, 1623, 1456, 1227, 1112, $1024 \mathrm{~cm}^{-1}$.

${ }^{1} \mathrm{H}$ NMR $\left(500 \mathrm{MHz}, \mathrm{CDCl}_{3}\right): \delta=8.06$ (br s, $\left.1 \mathrm{H}\right), 7.38-7.34(\mathrm{~m}, 5 \mathrm{H})$, 7.31-7.29 (m, $1 \mathrm{H}), 7.17-7.16(\mathrm{~m}, 1 \mathrm{H}), 7.14-7.13(\mathrm{~m}, 1 \mathrm{H}), 7.06-7.03$ $(\mathrm{m}, 1 \mathrm{H}), 4.87-4.66(\mathrm{~m}, 1 \mathrm{H}), 3.31-3.02(\mathrm{~m}, 2 \mathrm{H}), 2.52-2.30(\mathrm{~m}, 2 \mathrm{H})$.

${ }^{13} \mathrm{C}$ NMR $\left(126 \mathrm{MHz}, \mathrm{CDCl}_{3}\right): \delta=201.8,136.1,129.8,129.1,129.0$, 123.0, 122.8, 120.4, 120.2, 119.5, 119.0, 111.2, 111.1, 57.5, 45.1, 18.3 .

HRMS (ESI): $m / z[\mathrm{M}+\mathrm{Na}]^{+}$calcd for $\mathrm{C}_{18} \mathrm{H}_{15} \mathrm{NNaO}$ : 284.1051; found: 284.1053 .

\section{2-[2-(p-Tolyl)-1H-indol-3-yl]cyclobutanone (4l)}

Yield: $250 \mathrm{mg}$ (91\%); colorless oil.

FTIR (film): 3007, 1790, 1502, 1460, 1134, $1028 \mathrm{~cm}^{-1}$.

${ }^{1} \mathrm{H} \mathrm{NMR}\left(500 \mathrm{MHz}, \mathrm{CDCl}_{3}\right): \delta=8.07$ (br s, $\left.1 \mathrm{H}\right), 7.44(\mathrm{~d}, J=7.9 \mathrm{~Hz}, 1 \mathrm{H}$ ), $7.39(\mathrm{~d}, J=8.1 \mathrm{~Hz}, 2 \mathrm{H}), 7.37(\mathrm{~d}, J=8.1 \mathrm{~Hz}, 1 \mathrm{H}), 7.28$ (d, $J=7.9 \mathrm{~Hz}, 2 \mathrm{H})$, 7.21-7.17 (m, $1 \mathrm{H}), 7.13-7.09$ (m, $1 \mathrm{H}), 4.90-4.71(\mathrm{~m}, 1 \mathrm{H}), 3.35-3.12$ (m, $2 \mathrm{H}), 2.55-2.45(\mathrm{~m}, 2 \mathrm{H}), 2.42(\mathrm{~s}, 3 \mathrm{H})$.

${ }^{13} \mathrm{C}$ NMR $\left(126 \mathrm{MHz}, \mathrm{CDCl}_{3}\right): \delta=209.9,138.2,136.2,135.9,129.6$, 128.4, 127.7, 127.4, 122.3, 120.0, 119.3, 111.1, 107.6, 57.6, 45.0, 21.2, 18.3.

HRMS (ESI): $m / z[\mathrm{M}+\mathrm{Na}]^{+}$calcd for $\mathrm{C}_{19} \mathrm{H}_{17} \mathrm{NNaO}$ : 298.1208; found: 298.1211.

\section{2-(3-Methyl-1H-indol-2-yl)cyclobutanone (4m)}

Yield: $189 \mathrm{mg}$ (95\%); yellow oil.

FTIR (film): 3390, 3053, 2919, 1789, 1704, 1612, 1462, 1386, 1238 $\mathrm{cm}^{-1}$.
${ }^{1} \mathrm{H}$ NMR $\left(600 \mathrm{MHz}, \mathrm{CDCl}_{3}\right): \delta=7.49(\mathrm{dd}, J=7.9,3.5 \mathrm{~Hz}, 1 \mathrm{H}), 7.13(\mathrm{dd}$, $J=7.8,2.3 \mathrm{~Hz}, 2 \mathrm{H}$ ), 7.05 (ddd, $J=7.9,5.6,2.4 \mathrm{~Hz}, 1 \mathrm{H}$ ), 6.83 (d, $J=0.8$ $\mathrm{Hz}, 1 \mathrm{H}), 5.67-5.45(\mathrm{~m}, 1 \mathrm{H}), 3.11-3.03(\mathrm{~m}, 1 \mathrm{H}), 2.99-2.91(\mathrm{~m}, 1 \mathrm{H})$, $2.66(\mathrm{~d}, J=3.0 \mathrm{~Hz}, 1 \mathrm{H}), 2.62(\mathrm{ddd}, J=21.5,10.9,4.4 \mathrm{~Hz}, 1 \mathrm{H}), 2.22(\mathrm{~s}, 3$ $\mathrm{H})$.

${ }^{13} \mathrm{C}$ NMR (151 MHz, $\mathrm{CDCl}_{3}$ ): $\delta=203.6,135.8,128.9,122.8,121.9$, 119.4, 119.2, 112.2, 109.1, 68.5, 41.8, 20.2, 9.5.

HRMS (ESI): $m / z$ [M $+\mathrm{Na}]^{+}$calcd for $\mathrm{C}_{13} \mathrm{H}_{13} \mathrm{NNaO}: 222.0895$; found: 222.0901 .

3-[2-(2-0xocyclobutyl)-1H-indol-3-yl]propionic Acid Methyl Ester (4n)

Yield: $211 \mathrm{mg}$ (78\%); colorless oil.

FTIR (film): 3418, 2973, 2881, 1781, 1721, 1622, 1462, 1397, 1087, $912 \mathrm{~cm}^{-1}$.

${ }^{1} \mathrm{H}$ NMR $\left(600 \mathrm{MHz}, \mathrm{CDCl}_{3}\right): \delta=7.50(\mathrm{~d}, J=7.9 \mathrm{~Hz}, 1 \mathrm{H}), 7.13-7.10(\mathrm{~m}, 2$ H), 7.05 (ddd, $J=7.9,5.9,2.1 \mathrm{~Hz}, 1 \mathrm{H}), 6.86(\mathrm{~s}, 1 \mathrm{H}), 5.54(\mathrm{ddd}, J=10.5$, 4.1, $2.1 \mathrm{~Hz}, 1 \mathrm{H}), 3.59(\mathrm{~s}, 3 \mathrm{H}), 3.11-3.01(\mathrm{~m}, 1 \mathrm{H}), 2.99(\mathrm{t}, J=7.8 \mathrm{~Hz}, 2$ H), 2.97-2.90 (m, $1 \mathrm{H}), 2.60(\mathrm{t}, J=7.8 \mathrm{~Hz}, 2 \mathrm{H}), 2.59-2.56(\mathrm{~m}, 1 \mathrm{H})$, $2.26-2.18(\mathrm{~m}, 1 \mathrm{H})$.

${ }^{13} \mathrm{C}$ NMR (151 MHz, $\left.\mathrm{CDCl}_{3}\right): \delta=203.2,173.6,135.8,127.8,122.7$, 122.0, 119.6, 119.0, 115.2, 109.3, 68.4, 51.5, 41.8, 34.5, 20.4, 20.0.

HRMS (ESI): $m / z$ [M + Na] $]^{+}$calcd for $\mathrm{C}_{16} \mathrm{H}_{17} \mathrm{NNaO}_{3}$ : 294.1106; found: 294.1112.

\section{$N, N$-Diethyl-3-[2-(2-oxocyclobutyl)-1H-indol-3-yl]propionamide} (40)

Yield: $230 \mathrm{mg}$ (74\%); yellow oil.

FTIR (film): 3412, 2977, 2889, 1781, 1627, 1460, 1298, 1089, 1086 $\mathrm{cm}^{-1}$.

${ }^{1} \mathrm{H} \mathrm{NMR}\left(600 \mathrm{MHz}, \mathrm{CDCl}_{3}\right): \delta=7.52(\mathrm{~d}, J=7.9 \mathrm{~Hz}, 1 \mathrm{H}), 7.14-7.07(\mathrm{~m}, 2$ H), 7.04 (ddd, $J=8.0,4.9,3.1 \mathrm{~Hz}, 1 \mathrm{H}), 6.87(\mathrm{~s}, 1 \mathrm{H}), 5.55$ (ddd, $J=10.4$, 3.9, $2.0 \mathrm{~Hz}, 1 \mathrm{H}$ ), 3.30 (dd, $J=14.0,7.0 \mathrm{~Hz}, 2 \mathrm{H}$ ), 3.12 (dd, $J=14.2,7.1$ $\mathrm{Hz}, 2 \mathrm{H}$ ), 3.08-3.04 (m, $1 \mathrm{H}$ ), 3.02 (dd, $J=9.0,6.9 \mathrm{~Hz}, 2 \mathrm{H}), 2.93$ (dddd, $J=14.9,12.3,7.4,5.3 \mathrm{~Hz}, 1 \mathrm{H}), 2.63-2.59(\mathrm{~m}, 1 \mathrm{H}), 2.60-2.57(\mathrm{~m}, 2 \mathrm{H})$, $2.23(\mathrm{dt}, J=20.0,9.9 \mathrm{~Hz}, 1 \mathrm{H}), 1.02(\mathrm{t}, J=7.0 \mathrm{~Hz}, 3 \mathrm{H}), 0.99(\mathrm{t}, J=7.1 \mathrm{~Hz}$, $3 \mathrm{H})$.

${ }^{13} \mathrm{C}$ NMR (151 MHz, $\mathrm{CDCl}_{3}$ ): $\delta=203.3,171.7,135.7,127.9,122.8$, $121.9,119.5,119.0,115.9,109.3,68.4,41.8,41.7,40.1,33.6,20.8$, 19.9, 14.1, 12.9 .

HRMS (ESI): $m / z[\mathrm{M}+\mathrm{Na}]^{+}$calcd for $\mathrm{C}_{19} \mathrm{H}_{24} \mathrm{~N}_{2} \mathrm{NaO}_{2}$ : 335.1735; found: 335.1737.

\section{2-[3-(2-Nitro-1-phenylethyl)-1H-indol-2-yl]cyclobutanone (4p)}

Yield: $304 \mathrm{mg}$ (91\%); colorless oil.

FTIR (film): 2919, 1701, 1456, 1225, 1153, 907, $738 \mathrm{~cm}^{-1}$.

${ }^{1} \mathrm{H}$ NMR $\left(500 \mathrm{MHz}, \mathrm{CDCl}_{3}\right.$ ): $\delta=7.36$ (dd, $\left.J=18.2,8.0 \mathrm{~Hz}, 1 \mathrm{H}\right), 7.28-$ $7.22(\mathrm{~m}, 5 \mathrm{H}), 7.14(\mathrm{t}, J=4.1 \mathrm{~Hz}, 2 \mathrm{H}), 7.06-6.98(\mathrm{~m}, 1 \mathrm{H}), 6.92(\mathrm{~d}, J=$ $27.1 \mathrm{~Hz}, 1 \mathrm{H}), 5.59(\mathrm{dt}, J=17.0,5.3 \mathrm{~Hz}, 1 \mathrm{H}), 5.09(\mathrm{td}, J=7.9,4.5 \mathrm{~Hz}, 1$ H), 4.97 (ddd, $J=12.6,7.3,5.3 \mathrm{~Hz}, 1 \mathrm{H}$ ), 4.86 (ddd, $J=17.8,12.5,8.6 \mathrm{~Hz}$, $1 \mathrm{H}), 3.20-3.15$ (m, $1 \mathrm{H}), 2.98-2.95(\mathrm{~m}, 1 \mathrm{H}), 2.67-2.65$ ( $\mathrm{m}, 1 \mathrm{H}), 2.28-$ $24(\mathrm{~m}, 1 \mathrm{H})$.

${ }^{13} \mathrm{C}$ NMR $\left(126 \mathrm{MHz}, \mathrm{CDCl}_{3}\right): \delta=202.7,138.9,134.4,128.9,127.7$, 125.2, 124.3, 123.2, 122.9, 121.1, 118.9, 114.0, 109.4, 84.8, 79.4, 68.6, 27.5, 21.4 .

HRMS (ESI): $m / z[\mathrm{M}+\mathrm{Na}]^{+}$calcd for $\mathrm{C}_{20} \mathrm{H}_{18} \mathrm{~N}_{2} \mathrm{NaO}_{3}$ : 357.1215; found: 357.1221. 
2-[5-Methyl-3-(2-nitro-1-phenylethyl)-1H-indol-2-yl]cyclobutanone (4q)

Obtained as a 55:45 mixture of diastereoisomers.

Yield: $313 \mathrm{mg}$ (90\%); colorless oil.

FTIR (film): 3013, 2980, 2232, 1789, 1785, 1644, 1470, 1229, 1148, $1012 \mathrm{~cm}^{-1}$.

Isomer $\mathrm{A}$

${ }^{1} \mathrm{H}$ NMR $\left(500 \mathrm{MHz}, \mathrm{CDCl}_{3}\right): \delta=7.25-7.24(\mathrm{~m}, 4 \mathrm{H}), 7.20-7.17(\mathrm{~m}, 3 \mathrm{H})$, $7.00(\mathrm{~m}, 1 \mathrm{H}), 6.97-6.95(\mathrm{~s}, 1 \mathrm{H}), 5.53(\mathrm{~d}, J=8.2 \mathrm{~Hz}, 1 \mathrm{H}), 5.07-5.03(\mathrm{~m}$, $1 \mathrm{H}), 4.99-4.91(\mathrm{~m}, 1 \mathrm{H}), 4.90-4.80(\mathrm{~m}, 1 \mathrm{H}), 3.17-3.04(\mathrm{~m}, 1 \mathrm{H}), 3.03-$ 2.91 (m, $1 \mathrm{H}), 2.67-2.55$ (m, $1 \mathrm{H}), 2.31(\mathrm{~s}, 3 \mathrm{H}), 2.27-2.21(\mathrm{~m}, 1 \mathrm{H})$.

${ }^{13} \mathrm{C}$ NMR $\left(126 \mathrm{MHz}, \mathrm{CDCl}_{3}\right): \delta=202.6,138.9,134.4,129.7,129.0$, 128.9, 127.7, 127.5, 124.3, 122.9, 118.8, 114.0, 109.4, 79.4, 68.6, 28.5, 27.5, 21.3, 20.0.

HRMS (ESI): $m / z$ [M + Na] calcd for $\mathrm{C}_{21} \mathrm{H}_{20} \mathrm{~N}_{2} \mathrm{NaO}_{3}: 371.1372$; found: 371.1379 .

Isomer $B$ )

${ }^{1} \mathrm{H} \mathrm{NMR}\left(500 \mathrm{MHz}, \mathrm{CDCl}_{3}\right): \delta=7.25-7.23(\mathrm{~m}, 4 \mathrm{H}), 7.19-7.17(\mathrm{~m}, 2 \mathrm{H})$, $7.13-7.12(\mathrm{~m}, 1 \mathrm{H}), 7.02(\mathrm{~d}, J=4.5 \mathrm{~Hz}, 1 \mathrm{H}), 6.82(\mathrm{~s}, 1 \mathrm{H}), 6.66-6.23(\mathrm{~m}$, $1 \mathrm{H}), 5.07-5.04(\mathrm{~m}, 1 \mathrm{H}), 4.99-4.90(\mathrm{~m}, 1 \mathrm{H}), 4.89-4.80(\mathrm{~m}, 1 \mathrm{H}), 3.07-$ $3.06(\mathrm{~m}, 1 \mathrm{H}), 2.98-2.95(\mathrm{~m}, 1 \mathrm{H}), 2.76-2.74(\mathrm{~m}, 1 \mathrm{H}), 2.32(\mathrm{~s}, 3 \mathrm{H})$, $2.27-2.19(\mathrm{~m}, 1 \mathrm{H})$.

${ }^{13} \mathrm{C}$ NMR $\left(126 \mathrm{MHz}, \mathrm{CDCl}_{3}\right): \delta=202.7,138.9,134.4,129.7,129.0$, $128.9,127.7,125.2,122.9,121.1,118.9,114.0,109.4,84.9,68.7,41.9$, 28.4, 21.4, 20.1.

HRMS (ESI): $m / z[\mathrm{M}+\mathrm{Na}]^{+}$calcd for $\mathrm{C}_{21} \mathrm{H}_{20} \mathrm{~N}_{2} \mathrm{NaO}_{3}: 371.1372$; found: 371.1377.

\section{1-(5-Nitro-1H-indol-3-yl)cyclopropanecarbaldehyde (3r)}

Yield: $170 \mathrm{mg}$ (74\%); yellow solid.

All analytical data were in good accordance with reported data. ${ }^{16}$

\section{3-(1-Formylcyclopropyl)-1H-indole-4-carbonitrile (3s)}

Yield: $170 \mathrm{mg}$ (81\%); colorless oil.

All analytical data were in good accordance with reported data. ${ }^{16}$

\section{3-(1-Formylcyclopropyl)-1H-indole-5-carboxylic Acid Methyl Es- ter (3t)}

Yield: $218 \mathrm{mg}$ (90\%); colorless oil.

FTIR (film): 3472, 2961, 1785, 1711, 1620, 1437, 1261, $1096 \mathrm{~cm}^{-1}$.

${ }^{1} \mathrm{H}$ NMR $\left(600 \mathrm{MHz}, \mathrm{CDCl}_{3}\right): \delta=9.25(\mathrm{~s}, 1 \mathrm{H}), 8.30(\mathrm{~s}, 1 \mathrm{H}), 8.25(\mathrm{~s}, 1 \mathrm{H})$, $7.86(\mathrm{dd}, J=8.6,1.6 \mathrm{~Hz}, 1 \mathrm{H}), 7.30(\mathrm{~d}, J=8.6 \mathrm{~Hz}, 1 \mathrm{H}), 7.10(\mathrm{~d}, J=2.1 \mathrm{~Hz}$, $1 \mathrm{H}), 3.86(\mathrm{~s}, 3 \mathrm{H}), 1.61$ (q, J = 4.0 Hz, $2 \mathrm{H}), 1.35$ (q, J = 4.1 Hz, $2 \mathrm{H}$ ).

${ }^{13} \mathrm{C}$ NMR $\left(151 \mathrm{MHz}, \mathrm{CDCl}_{3}\right): \delta=201.1,168.0,138.8,127.4,125.4$, $123.9,122.1,121.9,114.8,111.1,51.8,28.7,15.8$

HRMS (ESI): $m / z$ [M $+\mathrm{Na}]^{+}$calcd for $\mathrm{C}_{14} \mathrm{H}_{13} \mathrm{NNaO}_{3}: 266.0793$; found: 266.0798 .

\section{3-(1-Formylcyclopropyl)-1H-indole-4-carboxylic Acid Methyl Es- ter (3u)}

Yield: $199 \mathrm{mg}$ (82\%); colorless oil.

All analytical data were in good accordance with reported data. ${ }^{16}$

3-(1-Formylcyclopropyl)-1H-indole-5-carbaldehyde (3v)

Yield: $155 \mathrm{mg}$ (73\%); colorless oil.
FTIR (film): 3348, 2960, 1726, 1665, 1611, 1258, $1094 \mathrm{~cm}^{-1}$. ${ }^{1} \mathrm{H}$ NMR $\left(600 \mathrm{MHz}, \mathrm{CD}_{3} \mathrm{OD}\right): \delta=9.94(\mathrm{~s}, 1 \mathrm{H}), 9.17(\mathrm{~s}, 1 \mathrm{H}), 8.09(\mathrm{~d}, J=$ $1.0 \mathrm{~Hz}, 1 \mathrm{H}), 7.72(\mathrm{dd}, J=8.5,1.5 \mathrm{~Hz}, 1 \mathrm{H}), 7.50(\mathrm{~d}, J=8.5 \mathrm{~Hz}, 1 \mathrm{H}), 7.33$ (s, $1 \mathrm{H}), 1.70(\mathrm{q}, J=3.8 \mathrm{~Hz}, 2 \mathrm{H}), 1.48-1.43(\mathrm{~m}, 2 \mathrm{H})$.

${ }^{13} \mathrm{C}$ NMR $\left(151 \mathrm{MHz}, \mathrm{CD}_{3} \mathrm{OD}\right): \delta=203.0,194.6,141.9,130.4,129.2$, $128.0,125.7,122.9,115.6,113.3,30.0,15.8$.

HRMS (ESI): $m / z$ [M + Na] $]^{+}$calcd for $\mathrm{C}_{13} \mathrm{H}_{11} \mathrm{NNaO}_{2}: 236.0687$; found: 236.0688 .

\section{1-(1-Benzyl-1H-pyrrol-2-yl)cyclopropanecarbaldehyde (3w)}

Obtained as an 80:20 mixture of regioisomers.

Yield: $153 \mathrm{mg}$ (68\%); colorless oil.

FTIR (film): 2998, 2982, 1728, 1660, 1224, 1048, 1002, $980 \mathrm{~cm}^{-1}$.

${ }^{1} \mathrm{H}$ NMR $\left(600 \mathrm{MHz}, \mathrm{CDCl}_{3}\right): \delta$ (major regioisomer) $=8.97(\mathrm{~s}, 1 \mathrm{H}), 7.23$ $(\mathrm{dt}, \mathrm{J}=12.1,2.9 \mathrm{~Hz}, 2 \mathrm{H}), 7.19-7.18(\mathrm{~m}, 2 \mathrm{H}), 6.88(\mathrm{~d}, \mathrm{~J}=1.8 \mathrm{~Hz}, 1 \mathrm{H})$, 6.67-6.59 (m, $1 \mathrm{H}), 6.11-6.07(\mathrm{~m}, 1 \mathrm{H}), 6.03(\mathrm{dt}, \mathrm{J}=12.9,6.4 \mathrm{~Hz}, 1 \mathrm{H})$, $4.98(\mathrm{~s}, 2 \mathrm{H}), 1.39$ (q, J = $3.9 \mathrm{~Hz}, 2 \mathrm{H}), 1.17$ (q, J = 4.0 Hz, $2 \mathrm{H}$ ).

${ }^{13} \mathrm{C}$ NMR (151 MHz, $\mathrm{CDCl}_{3}$ ): $\delta$ (major regioisomer) $=200.2,137.9$, 129.4, 128.7, 127.6, 126.9, 122.9, 110.5, 107.3, 50.9, 29.5, 16.8 .

HRMS (ESI): $m / z[\mathrm{M}+\mathrm{Na}]^{+}$calcd for $\mathrm{C}_{15} \mathrm{H}_{15} \mathrm{NNaO}$ : 248.1051; found: 248.1063.

\section{2-(2-Hydroxy-4,6-dimethoxyphenyl)cyclobutanone (4x)}

Yield: $190 \mathrm{mg}$ (86\%); colorless oil.

FTIR (film): 3412, 3023, 2988, 1787, 1462, 1228, 1144, $1021 \mathrm{~cm}^{-1}$.

${ }^{1} \mathrm{H}$ NMR $\left(500 \mathrm{MHz}, \mathrm{CDCl}_{3}\right): \delta=6.31(\mathrm{~s}, 1 \mathrm{H}), 6.07-5.96(\mathrm{~m}, 1 \mathrm{H}), 5.92$ $(\mathrm{d}, J=2.3 \mathrm{~Hz}, 1 \mathrm{H}), 4.74(\mathrm{dd}, J=10.7,8.3 \mathrm{~Hz}, 1 \mathrm{H}), 3.74(\mathrm{~s}, 3 \mathrm{H}), 3.71(\mathrm{~s}$, $3 \mathrm{H}), 3.16-3.02(\mathrm{~m}, 2 \mathrm{H}), 2.47-2.31(\mathrm{~m}, 1 \mathrm{H}), 2.20(\mathrm{dq}, J=10.5,8.5 \mathrm{~Hz}$, $1 \mathrm{H})$.

${ }^{13} \mathrm{C}$ NMR $\left(126 \mathrm{MHz}, \mathrm{CDCl}_{3}\right): \delta=213.2,160.2,159.1,155.2,105.2,94.1$, 91.3, 55.5, 55.2, 55.1, 44.1, 18.0 .

HRMS (ESI): $m / z$ [M + Na $]^{+}$calcd for $\mathrm{C}_{12} \mathrm{H}_{14} \mathrm{NaO}_{4}$ : 245.0790; found: 245.0799 .

\section{Funding Information}

We gratefully acknowledge funding from the Fondazione di Sardegna (CUP F71I17000180002).

\section{Supporting Information}

Supporting information for this article is available online at https://doi.org/10.1055/s-0040-1706087.

\section{References}

(1) (a) Gul, W.; Hamann, M. T. Life Sci. 2005, 78, 442. (b) KleinJúnior, L. C.; Cretton, S.; Vander Heyden, Y.; Gasper, A. L.; NejadEbrahimi, S.; Christen, P.; Henriques, A. T. J. Nat. Prod. 2020, 83, 852. (c) Tanifuji, R.; Minami, A.; Oguri, H.; Oikawa, H. Nat. Prod. Rep. 2020, 37, 109.

(2) (a) Humphrey, G. R.; Kuethe, J. T. Chem. Rev. 2006, 106, 2875. (b) Youn, S. W.; Ko, T. Y. Asian J. Org. Chem. 2018, 7, 1467. (c) Kumar, I.; Kumar, R.; Sharma, U. Synthesis 2018, 50, 2655. (d) Santos Neto, J. S.; Zeni, G. Org. Chem. Front. 2020, 7, 155

(3) Triggle, D. J.; Mitchell, J. M.; Filler, R. CNS Drug Rev. 1998, 4, 87. 
(4) Zhu, W.; Bao, X.; Ren, H.; Da, Y.; Wu, D.; Li, F.; Yan, Y.; Wang, L.; Chen, Z. Eur. J. Med. Chem. 2016, 115, 161.

(5) Anthes, R.; Benoit, S.; Chen, C.-K.; Corbett, E. A.; Corbett, R. M.; DelMonte, A. J.; Gingras, S.; Livingston, R. C.; Pendri, Y.; Sausker, J.; Soumeillant, M. Org. Process Res. Dev. 2008, 12, 178.

(6) Denya, I.; Malan, S. F.; Enogieru, A. B.; Omoruyi, S. I.; Ekpo, O. E.; Kapp, E.; Zindo, F. T.; Joubert, J. Med. Chem. Commun. 2018, 9, 357.

(7) Bogus, S. K.; Kuzmin, V. S.; Abramochkin, D. V.; Suzdalev, K. F.; Galenko-Yaroshevsky, P. A. J. Pharmacol. Sci. 2017, 133, 122.

(8) Fink, S. L.; Vojtech, L.; Wagoner, J.; Slivinski, N. S. J.; Jackson, K. J.; Wang, R.; Khadka, S.; Luthra, P.; Basler, C. F.; Polyak, S. J. Sci. Rep. 2018, 8, 8989.

(9) (a) Papahatjis, D. P.; Nikas, S.; Tsotinis, A.; Vlachou, M.; Makriyannis, A. Chem. Lett. 2001, 192. (b) Teng, X.; Degterev, A.; Jagtap, P.; Xing, X.; Choi, C.; Denu, R.; Yuan, J.; Cuny, G. D. Bioorg. Med. Chem. Lett. 2005, 15, 5039. (c) Piccio, P.; Vonhoff, S.; Davidson, K.; Teh, M.-T.; Sugden, D. J. Med. Chem. 2006, 49, 3509.

(10) Jandu, K. S.; Barrett, V.; Brockwell, M.; Cambridge, D.; Farrant, D. R.; Foster, C.; Giles, H.; Glen, R. C.; Hill, A. P.; Hobbs, H.; Honey, A.; Martin, G. R.; Salmon, J.; Smith, D.; Woollard, P.; Selwood, D. L. J. Med. Chem. 2001, 44, 681.

(11) Bartolozzi, A.; Chen, Z.; Dines, J. A.; Lo, H. Y.; Loke, P. L.; Olague, A.; Riether, D.; Tye, H.; Wu, L.; Zindell, R. M. US20130196967A1, 2013.

(12) Zhang, Y.; Yu, T.; Liu, B.; Zhang, X.; Zhang, S.; Cheng, C.; Zhang, J. WO2016037591A1, 2016.
(13) Goodacre, S. C.; Labadie, S.; Li, J.; Liang, J.; Liao, J.; Ortwine, D. F.; Ray, N. C.; Wang, X.; Zbieg, J.; Zhang, B. WO2017216279A1, 2017.

(14) (a) Harmata, M.; Ying, W.; Hong, X.; Barnes, C. L. Synthesis 2008, 4, 594. (b) Hu, H.; Wang, Y.; Qian, D.; Zhang, Z.-M.; Liu, L.; Zhang, J. Org. Chem. Front. 2016, 3, 759.

(15) Turnu, F.; Luridiana, A.; Cocco, A.; Porcu, S.; Frongia, A.; Sarais, G.; Secci, F. Org. Lett. 2019, 21, 7329.

(16) (a) Martis, A.; Luridiana, A.; Frongia, A.; Arca, M.; Sarais, G.; Aitken, D. J.; Guillot, R.; Secci, F. Org. Biomol. Chem. 2017, 15, 10053. (b) Porcu, S.; Luridiana, A.; Martis, A.; Frongia, A.; Sarais, G.; Aitken, D. J.; Boddaert, T.; Guillot, R.; Secci, F. Chem. Commun. 2018, 54, 13547. (c) Porcu, S.; Demuro, S.; Luridiana, A.; Cocco, A.; Frongia, A.; Aitken, D. J.; Charnay-Pouget, F.; Guillot, R.; Sarais, G.; Secci, F. Org. Lett. 2018, 20, 7699. (d) Secci, F.; Porcu, S.; Luridiana, A.; Frongia, A.; Ricci, P. C. Org. Biomol. Chem. 2020, 18, 3684.

(17) $\mathrm{N}$-Debenzylation of compound $\mathbf{4 f}$ was accomplished in a moderate yield (44\%) by using $\mathrm{NH}_{3} / \mathrm{Li}$ in THF at $-78^{\circ} \mathrm{C}$, as reported in the Supporting Information.

(18) (a) Conia, J. M.; Salaun, J. Acc. Chem. Res. 1972, 5, 33. (b) Barnier, J. P.; Denis, J. M.; Salaun, J.; Conia, J. M. Tetrahedron 1974, 30, 1397. (c) Gembus, V.; Karmazin, L.; Pira, S.; Uguen, D. Bull. Chem. Soc. Jpn. 2018, 91, 319.

(19) Brown, H. C.; Morgan, K. J.; Chloupek, F. J.J. Am. Chem. Soc. 1965, 87, 2137.

(20) Paquette, L. A.; Hofferberth, J. E. Org. React. 2003, 62, 477. 